\title{
Aberrantly regulated dysadherin and B-cell lymphoma 2/B-cell lymphoma 2-associated X enhances tumorigenesis and DNA targeting drug resistance of liver cancer stem cells
}

\author{
NAN JIANG ${ }^{1 *}$, WEI CHEN ${ }^{2 *}$, JIAN-WEN ZHANG ${ }^{1}$, YANG LI ${ }^{1}$, XIAN-CHENG ZENG ${ }^{1}$, \\ TONG ZHANG $^{1}$, BIN-SHENG FU ${ }^{1}$, HUI-MIN YI ${ }^{1}$ and QI ZHANG $^{1}$ \\ ${ }^{1}$ Department of Hepatic Surgery, The Third Affiliated Hospital of Sun Yat-sen University, Guangzhou, Guangdong 510630; \\ ${ }^{2}$ Department of Hepatopancreatobiliary Surgery, Second Affiliated Hospital, School of Medicine, \\ Zhejiang University, Hangzhou, Zhejiang 310009, P.R. China
}

Received November 15, 2014; Accepted August 4, 2015

DOI: $10.3892 / \mathrm{mmr} .2015 .4363$

\begin{abstract}
Cancer stem cells(CSCs) in hepatocellular carcinoma (HCC) are frequently resistant to current therapeutic regimens and therefore responsible for tumor recurrence. Previous studies have reported that expression levels of dysadherin in CSCs may be used as a prognostic indicator, which is also responsible for treatment failure and poor survival rates. The present study analyzed the association of enhanced dysadherin levels with drug resistance and evasion of apoptosis in human HCC SP cells. An SP of 3.7\% was isolated from human HCC cells using fluorescence-activated cell sorting. These SP cells displayed elevated levels of dysadherin and stemness proteins as well as high resistance to chemotherapeutic drugs and apoptosis. In order to reveal the possible link between dysadherin levels and tumorigenesis of SP cells, small interfering RNA technology was used to knockdown the expression of dysadherin in SP cells. Of note, the siRNA-transfected SP cells showed significantly reduced levels of stemness proteins, and were more sensitive to DNA-targeting drugs and apoptotic cell death as compared to non-transfected cells. Furthermore, in vivo experiments in NON/SCID mice indicated that dysadherin-expressing SP cells were highly tumorigenic, as they were able to induce tumor growth. The SP cell-derived tumor tissues in turn showed elevated dysadherin levels. The results of the present study therefore suggested that knockdown of
\end{abstract}

Correspondence to: Dr Nan Jiang, Department of Hepatic Surgery, The Third Affiliated Hospital of Sun Yat-sen University, 600 Tianhe Road, Tianhe, Guangzhou, Guangdong 510630, P.R. China

E-mail: njiang163@163.com

*Contributed equally

Key words: dysadherin, apoptosis, cancer stem cells, B-cell lymphoma-2, tumorigenesis dysadherin suppressed the tumorigenic properties of cancer stem-like SP cells. Hence, dysadherin is a valuable potential target for the development of novel anti-cancer drugs.

\section{Introduction}

Hepatocellular carcinoma (HCC) is the fifth most common cancer type and has a high mortality rate. During the past 20 years, there has been a net increase of $62 \%$ in HCC-associated annual mortality rates (from 463,000 to 752,000 ) (1). HCC is commonly diagnosed at the stage of metastasis to lungs, adrenal glands, lymph nodes and bones (2). After diagnosis and conventional treatments, the survival rate of individuals is poor due to the fact that HCC is highly resistant to chemoand radiotherapy $(3,4)$. The recently proposed cancer stem cell theory suggests the presence of a small distinct population of cancer cells named as 'cancer stem cells (CSCs)', which are responsible for minimal residual disease (MDR) after treatment failure (5). These CSCs possess the characteristics of normal stem cells, including high capacity for self-renewal as well as high differentiation, proliferation and cell survival (6). Hence, identification and characterization of CSCs will aid in the discovery of CSC-targeting agents for the complete elimination of MDR. The most commonly used and efficient method for the isolation of CSCs is the Hoechst 33342-dye exclusion method (7). This method is based on the fact that a small population of cancer cells expels the DNA-targeting dye due to the overexpression of adenosine triphosphate (ATP)-binding cassette $(\mathrm{ABC})$ transporter proteins; due to their separate appearance in fluorescence-activated cell sorting (FACS) dot plots, they are referred to as side population (SP) cells. SP cells are considered to contain high numbers of CSCs, as they display characteristic features of CSCs and are able to induce tumors in NOD/SCID mice even when administered at low cell concentrations (8).

Furthermore, a large number of studies on various cancer types reported that SP cells displayed an elevated expression of stem-cell surface markers, including CD133, CD44, EpCAM 
and Oct-4, which are responsible for high tumorigenesis and tumor invasion (9-11). Of note, recent clinical experimental studies reported that in CSCs, elevated expression of dysadherin (a membrane glycoprotein) has a vital role in cancer metastasis by upregulating the production of chemokines $(12,13)$. Previous studies on liver cancer cell lines also reported the overexpression of dysadherin in SP cells (13). These studies illustrated that reduced apoptosis and enhanced dysadherin in CSCs are linked to tumorigenesis and cancer metastasis; however, the molecular mechanisms of the regulatory functions of dysadherin in CSCs have remained elusive (14). In order to overcome the therapeutic complications in HCC and to develop novel anti-cancer drugs to target HCC stem cells, the phenotypic characterization of SP cells may be useful. Furthermore, it is known that deactivating mutations of the p53 gene and upregulation of B-cell lymphoma 2 (Bcl-2) lead to the evasion of apoptosis (15). Therefore, the present study analyzed the link between aberrant dysadherin expression and Bcl-2 signaling in SP cells with drug resistance and evasion of apoptosis in order to provide a basis for the development of CSC-specific therapeutic agents.

\section{Materials and methods}

Sample collection and cell culture. The hepatocellular carcinoma (HCC) samples were obtained from the patients at the time of surgery at the Department of Hepatic Surgery, The Third Affiliated Hospital of Sun Yat-sen University (Guangzhou, China). The tumor biopsies were obtained from the patients according to the legal and ethical guidelines approved by the The Third Affiliated Hospital of Sun Yat-sen University (Guangzhou, China). All patients provided written informed consent. The details of the patient cohort were as follows: Age range, 33-44; number of patients, 19; HCC grade, Edmondson-Steiner grade III; metastasis to lungs was present. The collected tumor samples were minced into fine fragments and cultured in $1 \mathrm{ml}$ fetal calf serum (FCS; Sigma-Aldrich, St. Louis MO, USA). In all assays the cells were obtained from all 19 patients. Cells were subsequently cultured in Dulbecco's modified Eagle's medium (DMEM; Sigma-Aldrich) with 10\% FCS, supplemented with antibiotics in T-75 flasks at $37^{\circ} \mathrm{C}$ in a humidified atmosphere of $5 \% \mathrm{CO}_{2}$ and $95 \%$ air. Upon becoming $90 \%$ confluent, cells were removed from the flasks using Trypsin-EDTA (0.25\%-53 mM EDTA; Sigma-Aldrich), washed, suspended in DMEM with $10 \%$ FCS and centrifuged at 4,500 x g for $6 \mathrm{~min}$. Cells were re-suspended in DMEM with $10 \%$ FCS. Cell counts were determined using a hemocytometer.

FACS analysis with Hoechst 33342 staining. The present study established the following experimental groups: Control, cells + Hoechst $3342(n=9$ samples from 9 patients); and drug-treated, cells + verapamil $($ Sigma-Aldrich $)+$ Hoechst $33342(n=9)$. Cells in staining medium $\left(10^{6}\right.$ cells/ml DMEM with $10 \%$ FCS $)$ were labeled with Hoechst 33342-bis-benzimide stock (final incubation concentration, $5 \mu \mathrm{l} / \mathrm{ml}$; Sigma-Aldrich) and optionally treated with verapamil (final incubation concentration, $0.8 \mu \mathrm{l} / \mathrm{ml}$ ). The cells were mixed and incubated in a water bath at $37^{\circ} \mathrm{C}$ for $90 \mathrm{~min}$. The cells were then centrifuged $\left(1,800 \mathrm{x} \mathrm{g}\right.$ for $10 \mathrm{~min}$ at $\left.4^{\circ} \mathrm{C}\right)$ and re-suspended in $500 \mu \mathrm{l}$ Hank's balanced salt solution (Sigma-Aldrich) containing 10 mM 4-(2-hydroxyethyl)-1-piperazineethanesulfonic acid (Sigma-Aldrich). Finally, the cells were counterstained with propidium iodide (PI; Sigma-Aldrich) $2 \mu \mathrm{g} / \mathrm{ml}$ at $4^{\circ} \mathrm{C}$. Cells were filtered through a $50-\mu \mathrm{m}$ nylon mesh (BD Biosciences, Franklin Lakes, NJ, USA) to remove cell clumps and filled into labeled FACS tubes. Separate tubes with medium (DMEM with $10 \%$ FCS) were kept for sterile sorting of SP cells and main population cells. The cells were sorted using a flow cytometer (FACS Aria II; BD Biosciences). The Hoechst 33342 dye was excited at $355 \mathrm{~nm}$ and its dual-wavelength fluorescence was analyzed (blue, $450 \mathrm{~nm}$; red, $675 \mathrm{~nm}$ ).

Reverse transcription quantitative polymerase chain reaction $(R T-q P C R)$ analysis. RNA was isolated by using the RNA isolation kit (Invitrogen Life Technologies, Inc., Carlsbad, CA, USA) and the RNA samples were treated with DNase I to exclude any DNA contamination. RT-qPCR were performed using the PrimeScript ${ }^{\mathrm{TM}}$ RT-PCR kit (Takara Bio, Inc., Otsu, Japan) and SYBR Premix Ex Taq II ${ }^{\mathrm{TM}}$ kit (Takara Bio, Inc.) according to the manufacturer's instructions, with the following thermocycling conditions using a T100 thermal cycler (Bio-Rad Laboratories, Inc., Hercules, CA, USA): $50^{\circ} \mathrm{C}$ for $60 \mathrm{~min}, 94^{\circ} \mathrm{C}$ for $5 \mathrm{~min}, 30$ cycles of $94^{\circ} \mathrm{C}$ for $30 \mathrm{sec}, 65^{\circ} \mathrm{C}$ for $30 \mathrm{sec}$ and $68^{\circ} \mathrm{C}$ for $60 \mathrm{sec}$. The following PCR primers from Shanghai Generay Biotech Co., Ltd (Shanghai, China) were used for RT-qPCR analysis: CD133 forward, 5'-TCTTG ACCGACTGAGAC-3' and reverse, 5'-ACTTGATGGATGCA CCAAGCAC-3'; GAPDH forward, 5'-TGGACTCCACGACG TACTCAG-3' and reverse, 5'-ACATGTTCCAATATGAT TCCA-3'; ABCG2 forward, 5'-AGCTGCAAGGAAAGATC CAA-3' and reverse, 5'-TCCAGACACACCACGGATAA-3'; OCT-4 forward, 5'-ATCCTGGGGGTTCTATTTGG-3' and reverse, 5'-CTCCAGGT TGCCTCTCACTC-3'; EрCAM forward, 5'-CTGCCAAATGTTTGGTGATG-3' and reverse, 5'-ACGCGTTGTGATCTCCTTCT-3'; BCL-2 forward, 5'-A CACTGTTAAGCATGTGCCG-3' and reverse, 5'-CCAGC TCATCTCACCTCACA-3'; BAX forward, 5'-GGATGCGT CCACCAAGAA-3' and reverse, 5'-ACTCCCGCCACAAAG ATG-3' (16-18). GAPDH was used as an internal control. Using a T100 thermal cycler, the thermocycling conditions were as follows: $-95^{\circ} \mathrm{C}$ for $2 \mathrm{~min}, 40$ cycles of $95^{\circ} \mathrm{C}$ for $30 \mathrm{sec}$, $55-60^{\circ} \mathrm{C}$ for $1 \mathrm{~min}$ and $72^{\circ}$ for $30-60 \mathrm{sec}$. PCR products were electrophoresed on a $1.2 \%$ agarose gel and stained with ethidium bromide. The gel was visualized using Bio-Rad ChemiDox XRS (Bio-Rad Laboratories, Inc.). The band intensity was measured by using Image J 1.0 software (National Institutes of Health, Bethesda, MD, USA), and relative gene expression was quantified using the $2^{-\Delta \Delta C T}$ method (19). The values presented in the graph are the average values of three independent experiments.

RNA interference. The small interfering RNA (siRNA) specific for dysadherin (GenBank accession no. AB072911) (17,18), was purchased from Dharmacon (Lafayette, CO, USA; cat no. 80026). siRNA transfection was performed according to the manufacturer's instructions (siRNA concentration of $200 \mathrm{nM}$ ). The transfected cells were analyzed after $48 \mathrm{~h}$ of incubation. 

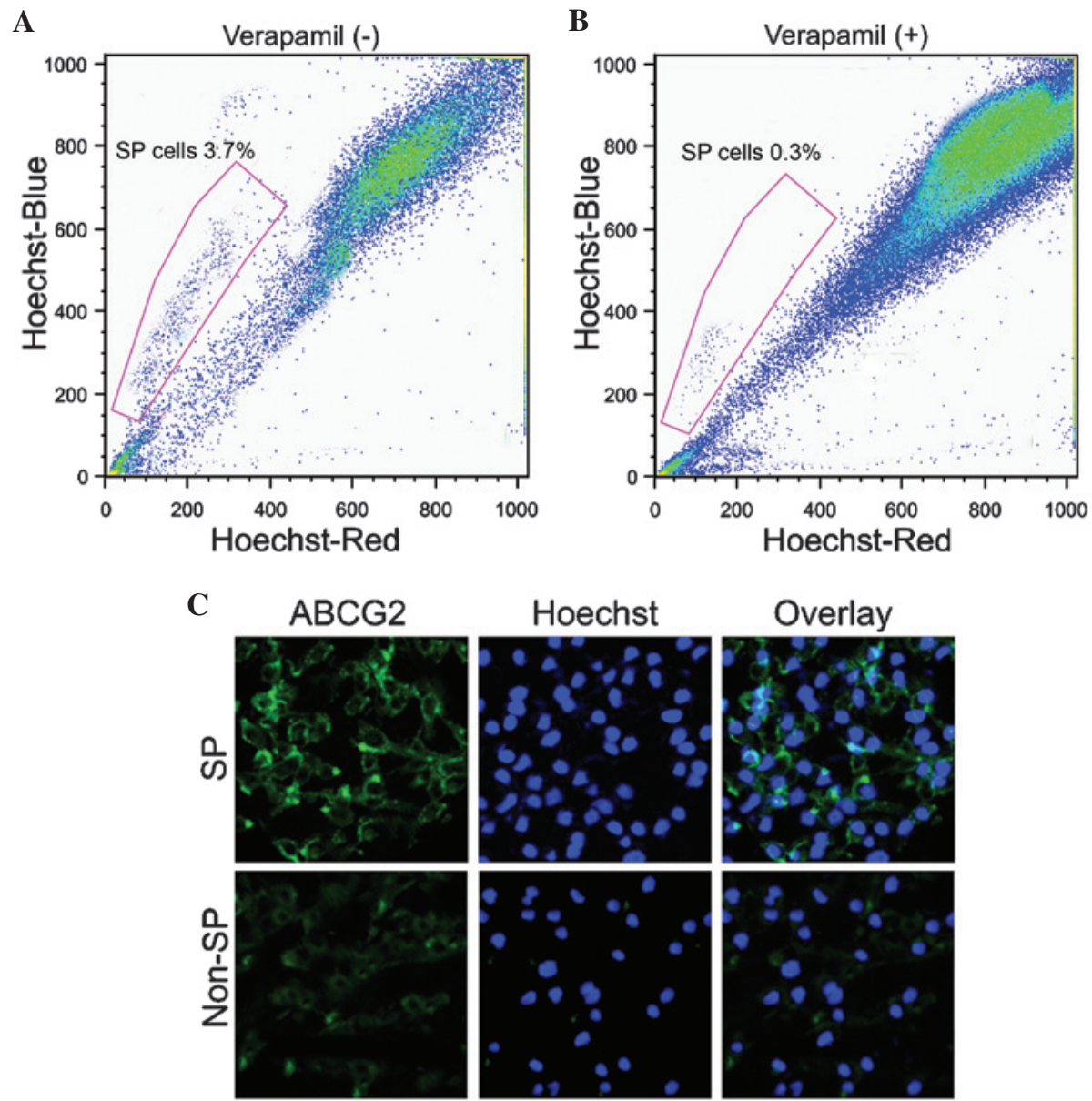

Figure 1. Identification of the SP of human hepatocellular carcinoma cells. (A) FACS dot plots showing the presence of an SP of 3.7\% (gated region), which was excluded from the main cell population using Hoechst 33342 staining. (B) The population of SP cells was significantly reduced to $0.3 \%$ after treatment with verapamil. (C) Immunocytochemical analysis of SP cells isolated by FACS showed higher levels of ABC transporter protein ABCG2 than non-SP cells. Magnification, x100. SP, side population; FACS, fluorescence-assisted cell sorting; ABCG2, adenosine triphosphate-binding cassette sub-family G member 2.

Western blot analysis. Protein was extracted from the SP and non-SP cells, and the protein concentration was determined using the Bradford assay (Pierce ${ }^{\mathrm{TM}}$ Coomassie protein assay kit; Invitrogen Life Technologies). Following 10\% SDS-PAGE and transfer onto a nitrocellulose membrane (Sigma-Aldrich), the membranes were incubated with primary antibodies overnight at $4^{\circ} \mathrm{C}$. The following primary antibodies were used: Mouse monoclonal anti-human ABCG2 (1:1,000; cat. no. sc-18841), rabbit polyclonal immunoglobulin (Ig)G dysadherin (1:1,000; cat. no. sc-98246) and mouse monoclonal anti-human GAPDH (1:1,000; cat. no. sc-47724). Secondary antibodies with alkaline phosphatase markers were used with specificity for the appropriate species: Goat anti-rabbit IgG (1:5,000; cat. no. sc-2034) and goat anti-mouse (1:5,000; cat. no. sc-2047), incubated for $2 \mathrm{~h}$ at room temperature. All antibodies were purchased from Santa Cruz Biotechnology, Inc., Dallas, TX, USA). Immunoreactive proteins were detected using a Chemiluminescence Reagent kit (cat. no. ab79907; Abcam, Cambridge, MA, USA). Blots were detected and scanned using a densitometer (GS-710; Bio-Rad Laboratories, Inc.).

Multidrug resistance assay. 3,000 cells/well in 96-well plates were seeded and cultured in DMEM/F12 (Sigma-Aldrich) supplemented with the necessary growth factors
(Sigma-Aldrich). After $7 \mathrm{~h}$ of incubation, SP and non-SP cells were treated with $5 \mu \mathrm{g} / \mathrm{ml}$ carboplatin (Sigma-Aldrich), $5 \mu \mathrm{g} / \mathrm{ml} 5$-flurouracil (5-FU; Sigma-Aldrich) and $5 \mu \mathrm{g} / \mathrm{ml}$ doxorubicin (Sigma-Aldrich). The mean optical density value at $450 \mathrm{~nm}\left(\mathrm{OD}_{450}\right)$ obtained was expressed as a graph. The drug resistance in the experimental groups was calculated using the following formula: Drug resistance rate $(\%)=\left(\mathrm{OD}_{450}\right.$ experimental group/ $\mathrm{OD}_{450}$ control group) $\mathrm{x} 100$.

Immunohisto/cytochemistry. The sorted SP cells and main population cells were seeded onto coverslips in 12-well plates $(100,000$ cells/well). After $24 \mathrm{~h}$ of incubation, the cells were rinsed with phosphate-buffered saline (PBS) and fixed in $4 \%$ paraformaldehyde in $1 \mathrm{X} \mathrm{PBS}$, for $15 \mathrm{~min}$ at room temperature. After washing with $1 \mathrm{X}$ PBS, cells were blocked with $1 \%$ bovine serum albumin (Sigma-Aldrich) in Tris-buffered saline (TBS; Sigma-Aldrich) with RNase (1:1,000 in 3\% BSA-TBS; Sigma-Aldrich). After $1 \mathrm{~h}$ of incubation at RT, cells were rinsed with PBS and incubated with the following primary antibodies (Santa Cruz Biotechnology, Inc.) in 1\% BSA-TBS: Mouse anti-ABC sub-family G member 2 (ABCG2; 1:1,000), mouse anti-Bcl2 (1:100); mouse anti-Bcl-2-associated X (Bax; 1:200) and mouse anti-dysadherin (1:1000), incubated overnight at $4^{\circ} \mathrm{C}$. After washing with $1 \mathrm{X}$ PBS, cells were incubated with secondary antibody conjugated to fluorescein isothiocyanate 
A

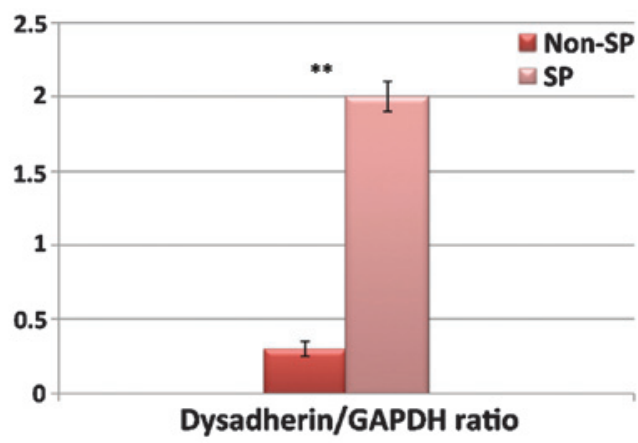

B
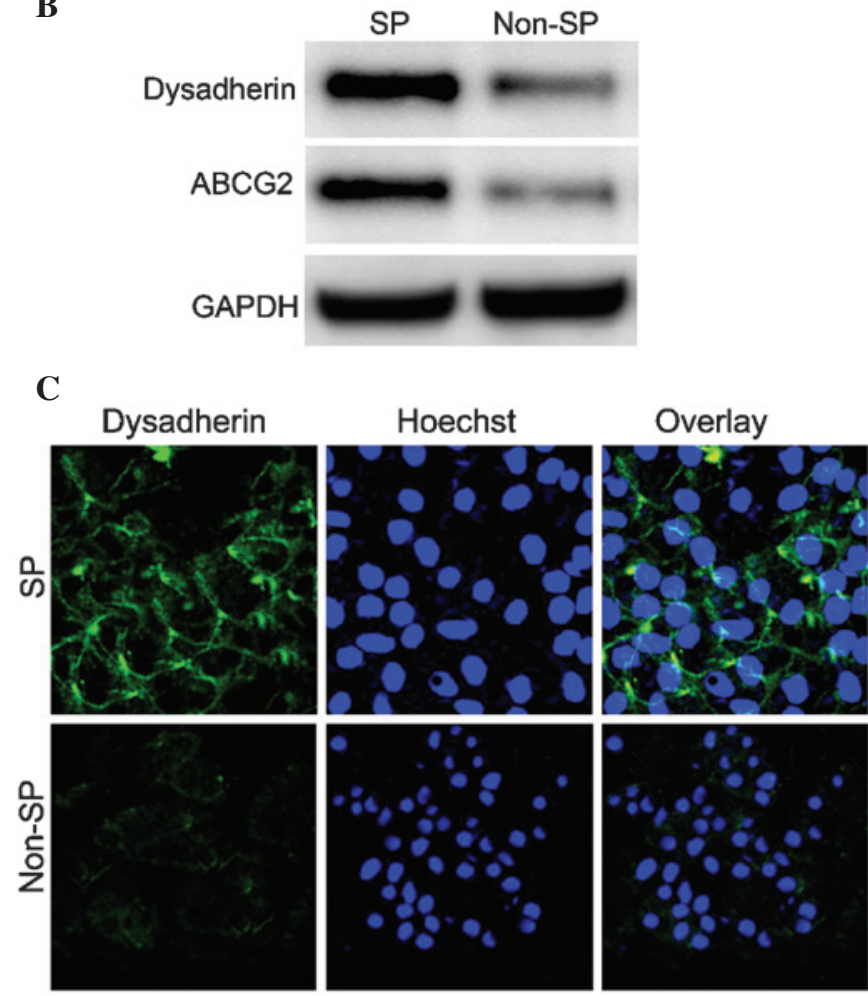

Figure 2. Enhanced expression of dysadherin in SP cells isolated by fluorescence-assisted cell sorting. (A) Reverse-transcription quantitative polymerase chain reaction analysis showing elevated mRNA expression of dysadherin in SP cells compared with that in non-SP cells. Values are expressed as the mean \pm standard deviation. ${ }^{* *} \mathrm{P}<0.01$ for $\mathrm{SP}$ vs. non-SP cells. (B) Western blot analysis showing increased dysadherin and ABCG2 expression in SP cells compared with that in non-SP cells. (C) Immunofluorescence analysis showed that dysadherin levels in SP cells were increased compared with those in non-SP cells. Magnification, x100. SP, side population; ABCG2, adenosine triphosphate-binding cassette sub-family G member 2 .

(dilution, 1:1000 in 1\% BSA-TBS) at room temperature for $1 \mathrm{~h}$. Following a further wash with PBS, Hoechst 33342 (Bio-Rad Laboratories, Inc.; dilution: 1:100) was used to stain the nuclei. For immunohistochemistry, the cells were processed and stained as described previously (20). The cells were viewed under a confocal laser scanning microscope (Leica TCS; Leica Microsystems, Oberkochen, Germany) and all the images were processed using Adobe Photoshop CS6 (Adobe Sytems, San Jose, CA, USA).

Tumor cell implantation. Mice (20 female and 20 male; age, six weeks-old; weight, 160-200 g) were purchased from the Experimental Animal Center of the Guangdong province
(Guangzhou, China). The mice were housed in plastic cages containing corn chip bedding and maintained on a $12-\mathrm{h}$ light-dark cycle (lights on at 7:00 a.m.), at 24-25 $\mathrm{C}$ and 50-60\% humidity, with access to food and water ad libitum. The mice sacrificed by injection of $75 \%$ alcohol. SP cells isolated by FACS or non-SP cells were mixed with Matrigel (Sigma-Aldrich) and subcutaneously inoculated into NOD/SCID mice (21). The density of cells injected and the tumor size were monitored according to the protocol of a previous study (21). The tumor volumes were calculated according to the formula $\mathrm{V}=1 / 2 \mathrm{ab}^{2}$ with a being the long diameter and $b$ the short diameter of the tumor. After 4-5 weeks, mice were sacrificed and tumors were harvested and measured, and their images were captured.

Statistical analysis. Values are expressed as the mean \pm standard deviation. One-way analysis of variance and Student's t-test were performed to determine significant differences between the treatment and control groups. SPSS 11.5 software (SPSS, Inc., Chicago, IL, USA) was used for statistical analysis. $\mathrm{P}<0.01$ was considered to indicate a statistically significant difference between values.

\section{Results}

SP cells are present in human HCC samples and overexpress $A B C$ transporters. The present study investigated cultured HCC cells from patients for the presence of SP cells using the Hoechst dye exclusion assay. As shown in Fig. 1A, a SP of $3.7 \%$ of the total cell population was detected in the HCC cells, whose presence was significantly diminished to $0.3 \%$ (Fig. 1B) after treatment with verapamil, an inhibitor of ABC transporters. Immunocytochemical analysis of the SP cells isolated by FACS showed enhanced expression of ABC transporter protein ABCG2 compared with that in non-SP cells (Figs. 1C and $2 \mathrm{~A}$ ). These results clearly indicated that overexpression of $\mathrm{ABC}$ transporter proteins in SP cells has a major role in resistance to chemotherapy by pumping the chemotherapeutic drugs out of the cells.

Dysadherin expression is enhanced in HCC SP cells. The HCC SP cells isolated by FACS were analyzed for the expression of dysadherin by RT-PCR. In SP cells, an increased expression of dysadherin mRNA compared with that in non-SP cells was identified (Fig. 2A). Western blot analysis showed that SP cells exhibited an elevated expression of dysadherin compared to that of non-SP cells (Fig. 2B). Furthermore, the elevated expression of dysadherin in SP cells was confirmed by immunocytochemistry (Fig. 2C).

Dysadherin enhances multidrug resistance and life span of SP cells. In line with the findings of previous studies $(13,17,18)$, the present study also reported elevated levels of dysadherin in SP cells (Fig. 2). Next, the present study investigated the role of dysadherin in SP cells by using siRNA technology in order to knockdown dysadherin expression in SP cells. First, the multi-drug resistance capacity of SP cells was tested prior to and after dysadherin knockdown. As shown in (Fig. 3A), SP cells showed a significantly higher survival rate $(>80 \%)$ following treatment with the chemotherapeutic drugs carboplatin, 5-flurouracil and doxorubicin as compared to that of non-SP 
A

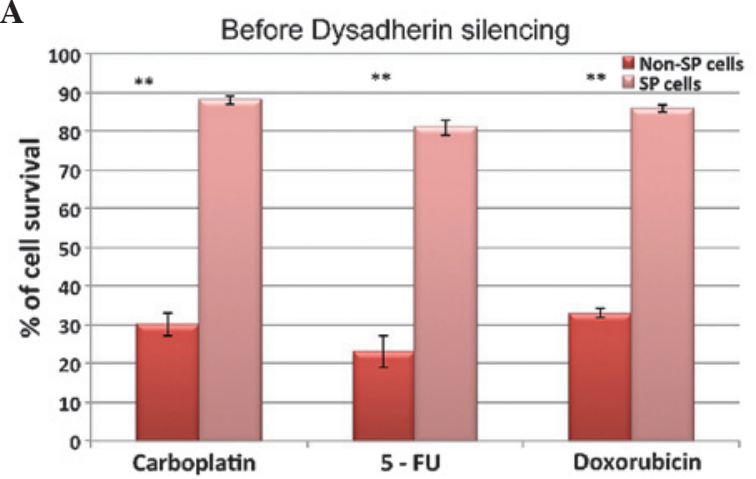

C

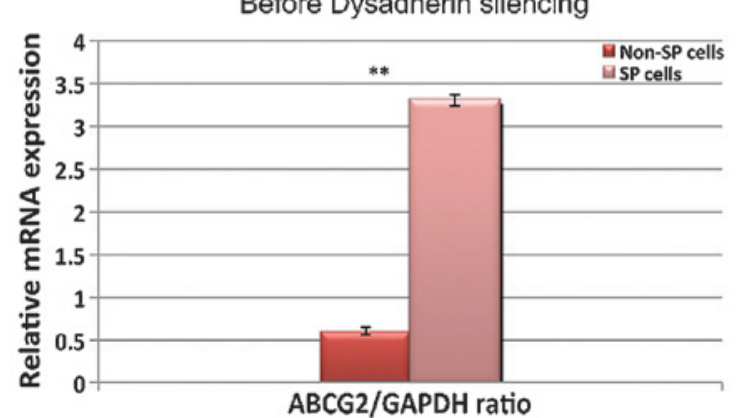

B

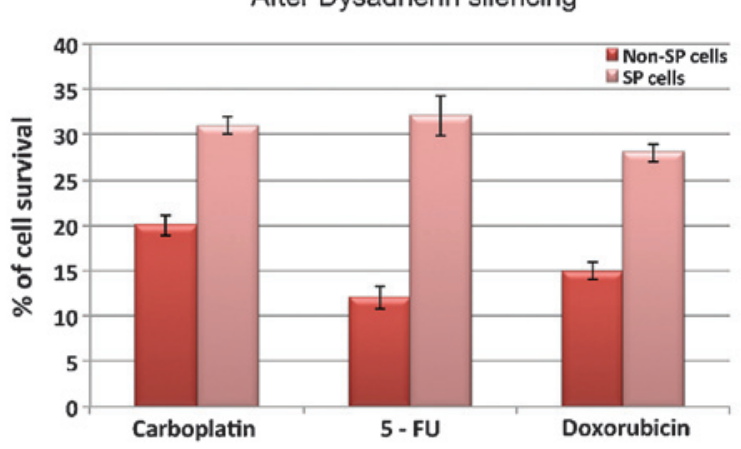

D

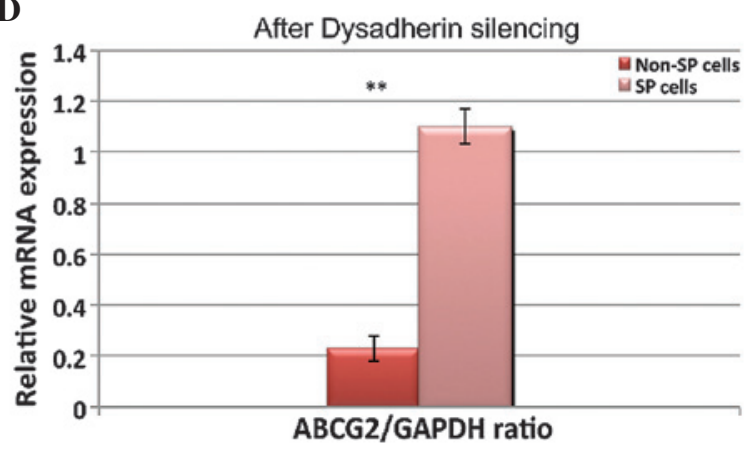

Figure 3. Comparison of cell survival rate of SP cells and non-SP cells treated with carboplatin, 5-FU or doxorubicin (A) before and (B) after dysadherin knockdown. (C and D) Before as well as after dysadherin silencing, the relative mRNA expression of ABCG2 in SP cells was significantly higher than in non-SP cells. Values are expressed as the mean \pm standard deviation. ${ }^{* *} \mathrm{P}<0.01$ for SP vs. non-SP cells. 5-FU, 5-flurouracil; SP, side population.

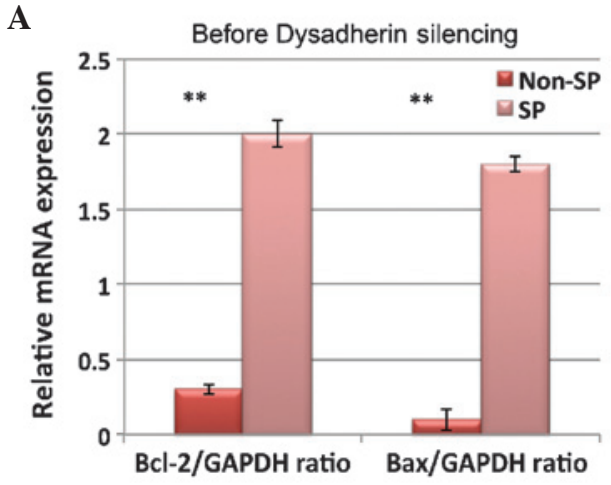

B
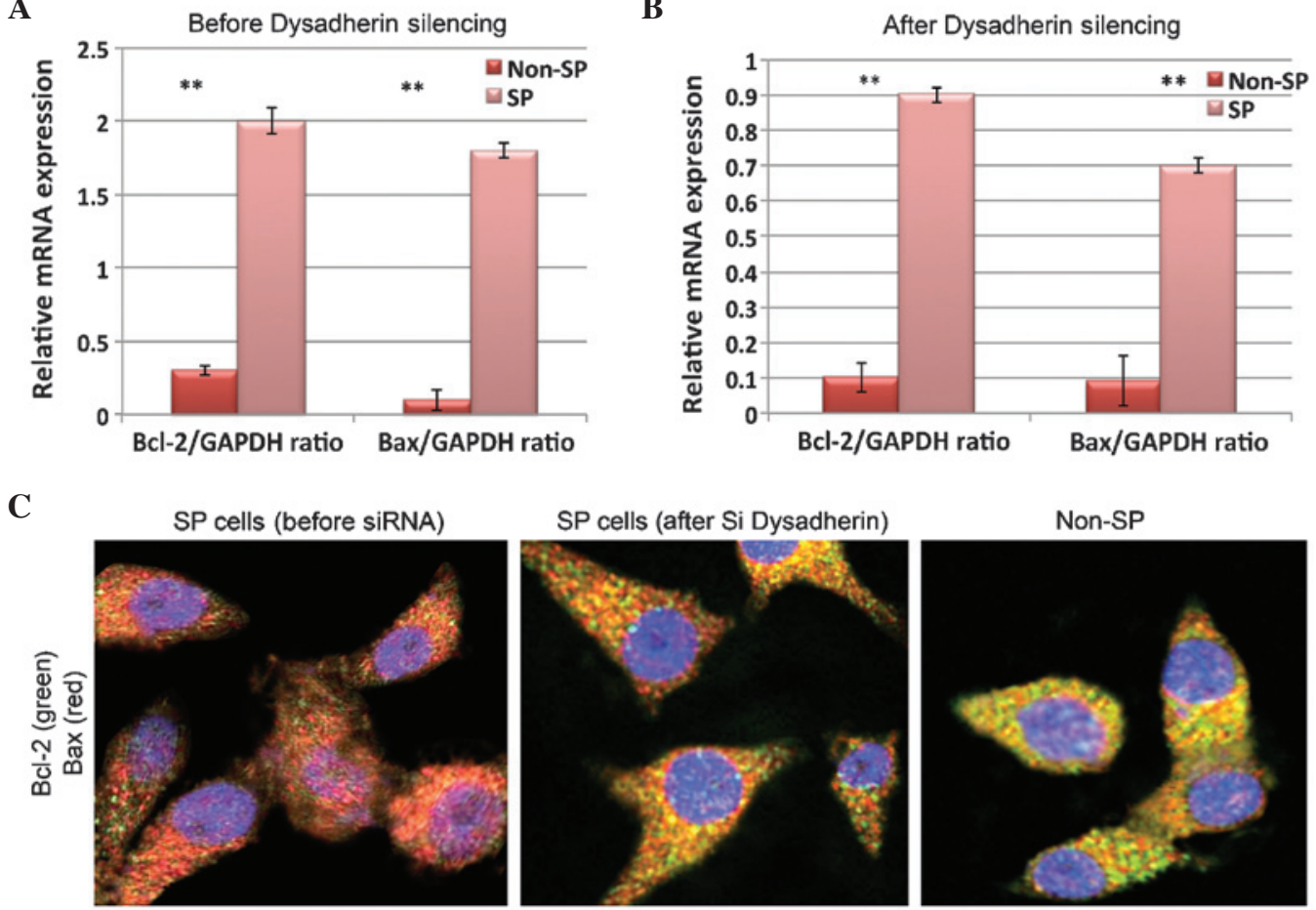

Figure 4. Relative mRNA expression of Bcl-2 and Bax (A) before and (B) after dysadherin knockdown (magnification, x100). Values are expressed as the mean \pm standard deviation. ${ }^{* *} \mathrm{P}<0.01$ for SP vs. non-SP cells. (C) Fluorescence microscopic analysis of Bcl-2 (red) and Bax (green) expression in SP cells and non-SP cells before and after dysadherin-specific siRNA interference (magnification, x120). Bcl-2, B-cell lymphoma 2; Bax, Bcl-2-associated X; siRNA, small interfering RNA; SP, side population.

cells. However, after dysadherin knockdown, the survival rate of SP cells upon treatment with the abovementioned drugs was markedly reduced (Fig. 3B). Of note, the relative mRNA expression of $\mathrm{ABC}$ transporter gene $\mathrm{ABCG} 2$ was also significantly reduced after silencing of dysadherin compared with that prior to silencing (Fig. 3C and D). These results suggested that 
A

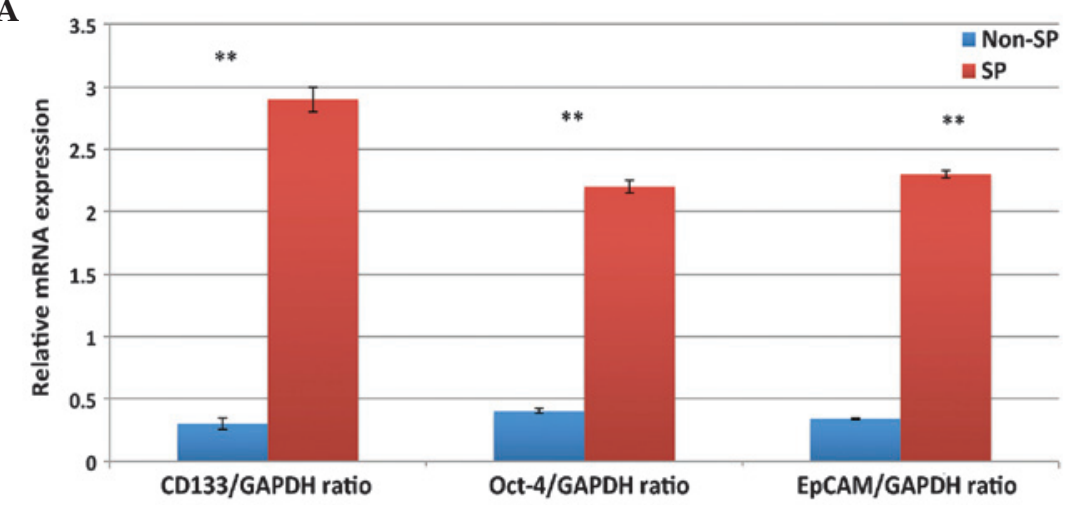

B

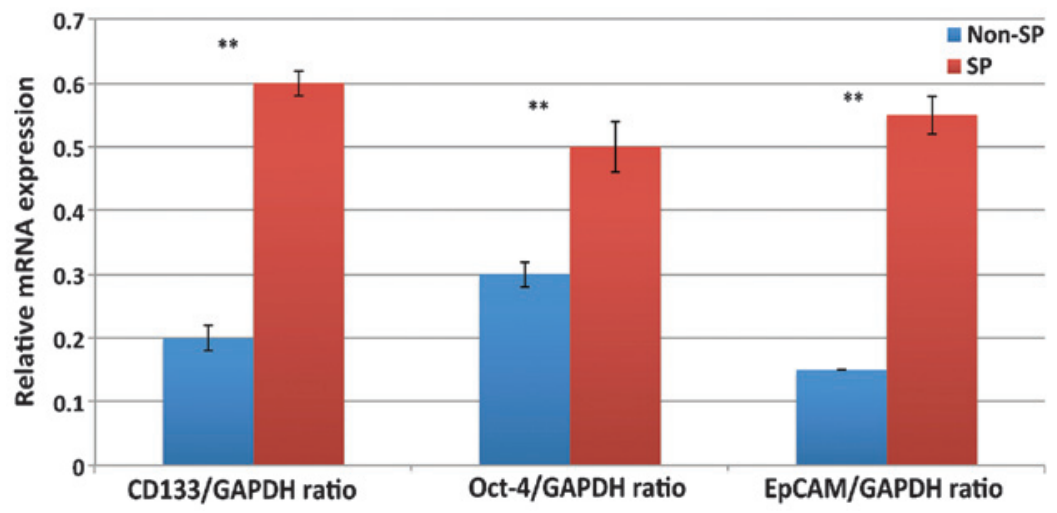

Figure 5. Comparison of mRNA expression levels of stemness genes CD133, OCT-4 and EpCAM (A) before and (B) after dysadherin knockdown in SP cells. Values are expressed as the mean \pm standard deviation. ${ }^{* * *} \mathrm{P}<0.01$ for $\mathrm{SP}$ vs. non-SP cells. SP, side population.

\section{A}

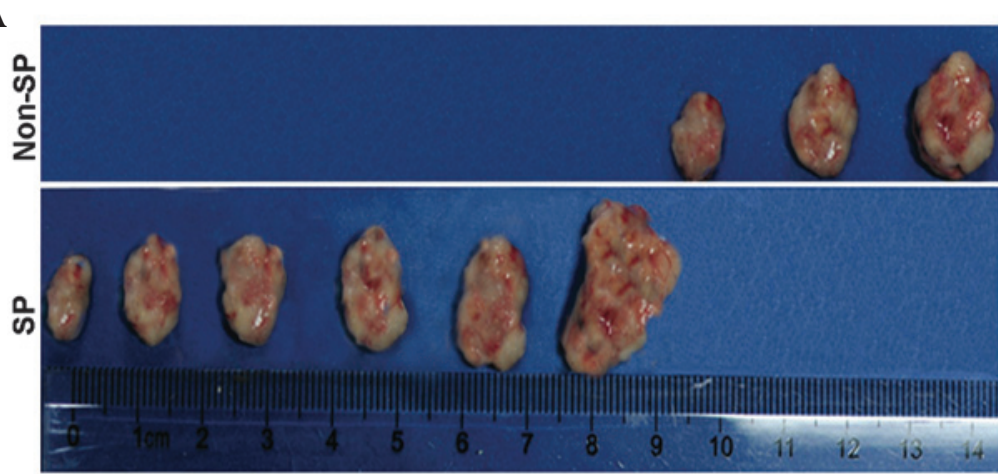

B

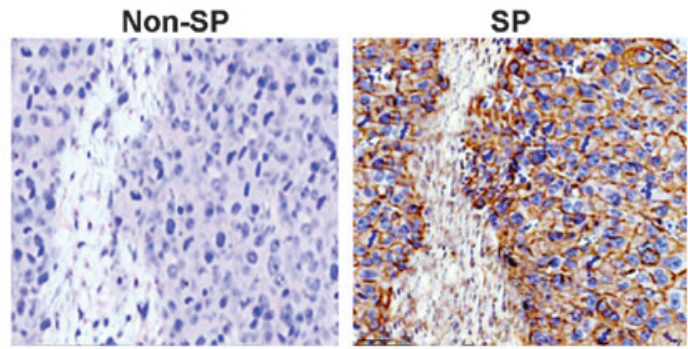

Figure 6. SP cells of hepatocellular carcinoma cells are highly tumorigenic. (A) Tumor sections derived from SP and non-SP cells after 23 days of inoculation into NOD/SCID mice. (B) Immunohistochemical staining for dysadherin in tumor tissues of SP and non-SP cells. Magnification, x100. SP, side population.

dysadherin has a major role in drug resistance of SP cells, either by direct or indirect regulation of $\mathrm{ABCG} 2$.

Dysadherin reduces apoptotic cell death in HCC SPs. Since the silencing of dysadherin efficiently reduced the survival rate of SP cells after drug treatment (Fig. 3), it was speculated whether the dysadherin was involved in regulation of apoptosis in SP cells as well. RT-PCR analysis showed that mRNA expression of anti-apoptotic gene $\mathrm{Bcl}-2$ and pro-apoptotic Bax was upregulated in SP cells compared with that in 
non-SP cells (Fig. 4A). Of note, the dysadherin-silenced SP cells exhibited reduced expression of Bcl-2 and Bax compared with that prior to silencing (Fig. 4B). However, immunocytochemistry indicated that Bcl-2 was decreased and Bax was elevated in SP cells compared with those in non-SP cells, while dysadherin-knockdown led to an increase in Bcl-2 and a decrease in Bax (Fig. 4C). These results indicate that dysadherin affects Bcl-2 and Bax mRNA expression differently from their protein expression; however, the protein expression levels indicated that dysadherin overexpression may, due to upregulating Bcl-2 and downregulating Bax, be associated with drug resistance and evasion of apoptosis in HCC SP cells.

Dysadherin knockdown reduces the expression of stemness genes in SP cells. It is well known that SP cells are have a high capacity for self-renewal and are able to generate an increased number of tumor spheres due to overexpressing stemness genes (22). Therefore, the present study evaluated the expression of stemness genes in SP cells prior to and after dysadherin knockdown. After silencing, the transcription of the stemness genes CD133, Oct-4 and EpCAM, which are essential for the maintenance of CSC self-renewal, was significantly reduced (Fig. 5A and B).

HCC SP cells are highly tumorigenic. Next, the present study performed a tumor xenograft experiment in order to evaluate the tumorigenic potential of SP cells. The results showed that SP cells were able to induce tumor growth in NOD/SCID mice, even when inoculated at low cell numbers (i.e., 5,000); however, the non-SP cells failed to induce massive tumor growth at this cell density (data not shown). Compared with the non-SP cell-derived tumors, the SP cell-derived tumors grew faster and displayed increased sizes (Fig. 6A). Immunohistochemical staining also confirmed that SP cell-derived tumor tissues showed increased dysadherin staining compared with non-SP tumors (Fig. 6B).

\section{Discussion}

$\mathrm{HCC}$ is one of the major leading causes of cancer-associated mortality worldwide (23). Clinical and experimental studies reported that the presence of CSCs in HCC has a major role in chemotherapy resistance, tumor relapse and metastasis (24). Cancer stem-like SP cells have been identified and characterized in several cancer cell lines, including the MHCC97 HCC cell line, and it has been indicated that SP cells are highly resistant to chemotherapy $(16,22,25-27)$. In addition, SP cells showed elevated expression of ABC transporter proteins, which are the main cause of drug resistance by active drug efflux (28-30). However, to date, the underlying molecular mechanisms and signaling pathways of the regulation of $\mathrm{ABC}$ transporter overexpression and multi-drug resistance have remained to be fully elucidated. There may be an indirect link between ABC transporters and apoptotic pathways, which jointly contribute to the capacity of SP cells for drug resistance and evasion of apoptosis resistance.

Elevated dysadherin and its significance in cancer metastasis has been reported in SP cells of HCC cell lines $(17,31)$ Dysadherin, a member of the FXYD family, regulates
NaK-ATPase, which is in turn actively involved in cell permeability and polarity and is essential for cell signaling and stem cell dynamics (32-34). The present study reported that SP cells from human HCC displayed increased expression of dysadherin together with elevated expression of ABCG2 and the stemness genes CD133, Oct-4 and EpCAM. Following knockdown of dysadherin by RNA interference, the SP cells displayed increased sensitivity to chemotherapeutic drugs, as the expression of drug resistance-associated ABCG2 was inhibited. It is therefore speculated that dysadherin may also regulate the expression of other multidrug resistance genes, including ABCA1 and ABCB1. Furthermore, following dysadherin knockdown, SP cells displayed enhanced levels of pro-apoptotic protein (Bax) as well as downregulated expression of stemness genes, suggesting that dysadherin has a crucial role in SP-cell maintenance and survival. Similar to the present findings, it was previously shown that dysadherin has a major role in the maintenance of the CSC-like properties of SP cells, while its knockdown efficiently reduced CSC-like characteristics (17). In the present study, the dysadherin-overexpressing SP cells were shown to have a high potential for self-renewal and to be highly tumorigenic. The present study also evidenced that dysadherin-overexpressing SP cells were able to induce tumor growth in NOD/SCID mice and the tumor tissues derived from the mice were highly positive for dysadherin staining.

The key regulator of apoptosis is p53, which is linked to numerous pro-apoptotic genes, including Bcl-2 (anti-apoptotic factor) and Bax. Previous studies on liver cancer cell lines showed that the expression of Bcl-2 and Bax in SP cells was aberrantly altered and therefore, the rate of apoptosis was markedly reduced in SP cells (16). Bcl-2 is an anti-apoptotic protein whose enhanced expression inhibits Bax, which would otherwise provoke the release of cytochrome $c$ from the outer mitochondrial membrane protein, which in turn leads to the formation of apoptosome and caspase activation (35). It is difficult to conclude based on the present study whether the altered ratio of Bcl-2/Bax may lead to lower drug sensitivity. It has previously been reported that Bcl-2 is able to inhibit Bax (35). It is possible that upregulated Bax expression is caused by downregulated Bcl-2 expression, mediated by dysadherin.

The present study showed that the protein expression in Bcl-2 was upregulated and that of Bax was downregulated in HCC SP cells, therefore indicating that SP cells have an enhanced survival rate compared to that of non-SP cells, even after treatment with chemotherapeutic drugs. These results indicated that dysadherin has a pivotal role in drug resistance and evasion of apoptosis in SP cells and thereby, SP cells may be spared by chemotherapy and tumor recurrence may occur.

In conclusion, the results of the present study suggested that dysadherin may enhance the expression of drug efflux pumps and anti-apoptotic mechanisms in SP cells either by direct or indirect interaction. However, the precise underlying molecular mechanism of dysadherin-mediated tumorigenesis remains to be elucidated in future studies. However, the results of the present study indicated that novel anti-cancer drugs targeting the transcriptional regulation of dysadherin may efficiently suppress the activity and drug resistance of SP cells. 


\section{Acknowledgements}

The present study was supported by the National Natural Science Foundation of China (grant nos. 81000959 and 81201781), the Guangdong Natural Science Foundation (grant no. S2013010016023), the Science and Technology Program of Guangzhou (grant no. 2014A020212084), the Fundamental Research Funds for the Central Universities (grant no. 12ykpy47), and the National 12th Five-Year Science and Technology Plan Major Projects of China (grant no. 2012ZX10002017-005).

\section{References}

1. Forner A, Llovet JM and Bruix J: Hepatocellular carcinoma. Lancet 379: 1245-1255, 2012

2. Bruix J and Sherman M: Management of hepatocellular carcinoma. Hepatology 42: 1208-1236, 2005.

3. Farazi PA and DePinho RA: Hepatocellular carcinoma pathogenesis: From genes to environment. Nat Rev Cancer 6: 674-687, 2006.

4. Lee TK, Castilho A, Ma S and Ng IO: Liver cancer stem cells: Implications for a new therapeutic target. Liver Int 29: 955-965, 2009.

5. Yu Z, Pestell TG, Lisanti MP and Pestell RG: Cancer stem cells. Int J Biochem Cell Biol 44: 2144-2151, 2012.

6. Gupta PB, Chaffer CL and Weinberg RA: Cancer stem cells: Mirage or reality? Nat Med 15: 1010-1012, 2009.

7. Oates JE, Grey BR, Addla SK, Samuel JD, Hart CA, Ramani VA, Brown MD and Clarke NW: Hoechst 33342 side population identification is a conserved and unified mechanism in urological cancers. Stem Cells Dev 18: 1515-1522, 2009.

8. Suetsugu A, Nagaki M, Aoki H, Motohashi T, Kunisada T and Moriwaki H: Characterization of CD133+ hepatocellular carcinoma cells as cancer stem/progenitor cells. Biochem Biophys Res Commun 351: 820-824, 2006.

9. Al-Hajj M, Wicha MS, Benito-Hernandez A, Morrison SJ and Clarke MF: Prospective identification of tumorigenic breast cancer cells. Proc Natl Acad Sci USA 100: 3983-3988, 2003.

10. Patrawala L, Calhoun-Davis T, Schneider-Broussard R and Tang DG: Hierarchical organization of prostate cancer cells in xenograft tumors: The CD44+alpha2-beta1+ cell population is enriched in tumor-initiating cells. Cancer Res 67: 6796-6805, 2007.

11. Dalerba P, Dylla SJ, Park IK, Liu R, Wang X, Cho RW, Hoey T, Gurney A, Huang EH, Simeone DM, et al: Phenotypic characterization of human colorectal cancer stem cells. Proc Natl Acad Sci USA 104: 10158-10163, 2007.

12. Ino Y, Gotoh M, Sakamoto M, Tsukagoshi K and Hirohashi S: Dysadherin, a cancer-associated cell membrane glycoprotein, down-regulates E-cadherin and promotes metastasis. Proc Natl Acad Sci USA 99: 365-370, 2002.

13. Nam JS, Hirohashi S and Wakefield LM: Dysadherin: A new player in cancer progression. Cancer Lett 255: 161-169, 2007.

14. Qin LX and Tang ZY: Recent progress in predictive biomarkers for metastatic recurrence of human hepatocellular carcinoma: A review of the literature. J Cancer Res Clin Oncol 130: 497-513, 2004.

15. Royds JA and Iacopetta B: p53 and disease: When the guardian angel fails. Cell Death Differ 13: 1017-1026, 2006.

16. Fan J, Li R, Zhang R, Liu HL, Zhang N, Zhang FQ and Dou KF: Effect of Bcl-2 and Bax on survival of side population cells from hepatocellular carcinoma cells. World J Gastroenterol 13: 6053-6059, 2007.
17. Park JR, Kim RJ, Lee YK, Kim SR, Roh KJ, Oh SH, Kong G, Kang KS and Nam JS: Dysadherin can enhance tumorigenesis by conferring properties of stem-like cells to hepatocellular carcinoma cells. J Hepatol 54: 122-131, 2011.

18. Shimamura T, Yasuda J, Ino Y, Gotoh M, Tsuchiya A, Nakajima A, Sakamoto M, Kanai Y and Hirohashi S: Dysadherin expression facilitates cell motility and metastatic potential of human pancreatic cancer cells. Cancer Res 64 : 6989-6995, 2004.

19. Livak KJ and Schmittgen TD: Analysis of relative gene expression data using real-time quantitative PCR and the 2(-Delta Delta (CT)) Method. Methods 25: 402-408, 2001.

20. Kim RJ, Kim SR, Roh KJ, Park SB, Park JR, Kang KS, Kong G, Tang B, Yang YA, Kohn EA, et al: Ras activation contributes to the maintenance and expansion of Sca-1pos cells in a mouse model of breast cancer. Cancer Lett 287: 172-181, 2010

21. Shi Y, Fu X, Hua Y, Han Y, Lu Y and Wang J: The side population in human lung cancer cell line NCI-H460 is enriched in stem-like cancer cells. PLoS One 7: e33358, 2012.

22. Patrawala L, Calhoun T, Schneider-Broussard R, Zhou J, Claypool K and Tang DG: Side population is enriched in tumorigenic, stem-like cancer cells, whereas ABCG2+ and ABCG2-cancer cells are similarly tumorigenic. Cancer Res 65: 6207-6219, 2005

23. Bosch FX, Ribes J and Borràs $\mathrm{J}$ : Epidemiology of primary liver cancer. Semin Liver Dis 19: 271-285, 1999.

24. Lee TK, Castilho A, Cheung VC, et al: CD24(+) liver tumorinitiating cells drive self-renewal and tumor initiation through STAT3-mediated NANOG regulation.Cell Stem Cell9:50-63, 2011.

25. Szotek PP, Pieretti-Vanmarcke R, Masiakos PT, Dinulescu DM, Connolly D, Foster R, Dombkowski D, Preffer F, Maclaughlin DT and Donahoe PK: Ovarian cancer side population defines cells with stem cell-like characteristics and mullerian inhibiting substance responsiveness. Proc Natl Acad Sci USA 103: 11154-11159, 2006.

26. Haraguchi N, Utsunomiya T, Inoue H, Tanaka F, Mimori K, Barnard GF and Mori M: Characterization of a side population of cancer cells from human gastrointestinal system. Stem Cells 24: 506-513, 2006.

27. Hirschmann-Jax C, Foster AE, Wulf GG, Goodell MA and Brenner MK: A distinct 'side population' of cells in human tumor cells: Implications for tumor biology and therapy. Cell Cycle 4: 203-205, 2005.

28. Bunting KD, Zhou S, Lu T and Sorrentino BP: Enforced P-glycoprotein pump function in murine bone marrow cells results in expansion of side population stem cells in vitro and repopulating cells in vivo. Blood 96: 902-909, 2000.

29. Visvader JE and Lindeman GJ: Cancer stem cells in solid tumours: Accumulating evidence and unresolved questions. Nat Rev Cancer 8: 755-768, 2008.

30. Norwood K, Wang RY, Hirschmann-Jax C, Andreeff M, Brenner MK, Goodell MA and Wulf GG: An in vivo propagated human acute myeloid leukemia expressing ABCA3. Leuk Res 28: 295-299, 2004

31. Nam JS, Hirohashi S and Wakefield LM: Dysadherin: A new player in cancer progression. Cancer Lett 255: 161-169, 2007.

32. Garty $\mathrm{H}$ and Karlish SJ: Role of FXYD proteins in ion transport. Annu Rev Physiol 68: 431-459, 2006.

33. Rajasekaran SA and Rajasekaran AK: Na, K-ATPase and epithelial tight junctions. Front Biosci (Landmark Ed) 14: 2130-2148, 2009.

34. Shin K, Fogg VC and Margolis B: Tight junctions and cell polarity. Annu Rev Cell Dev Biol 22: 207-235, 2006.

35. Korsmeyer SJ,Shutter JR, Veis DJ, Merry DE and Oltvai ZN: Bcl-2/Bax: A rheostat that regulates an anti-oxidant pathway and cell death. Semin Cancer Biol 4: 327-332, 1993. 